\title{
Reversible magnetism switching in graphene-based systems via the decoration of photochromic molecules
}

\author{
Argo Nurbawono ${ }^{1}$,Chun Zhang ${ }^{1,2 *}$ \\ ${ }^{1}$ Department of Physics and Graphene Research Centre, \\ National University of Singapore, 2 Science Drive 3, Singapore, 117542 \\ ${ }^{2}$ Department of Chemistry, National University of Singapore, \\ 3 Science Drive 3, Singapore 117543
}

(Dated: August 26, 2018)

\begin{abstract}
By first principles calculations, we demonstrate that when decorated with photochromic molecules, it is possible to use light to reversibly control the magnetic properties of a nanoscale magnetic system. The combination of a graphene-based magnetic system and a photochromic azobenzene molecule is chosen as a model system. The trans and cis isomers of the azobenzene molecule that can be converted between each other by means of photoexcitations are found to have drastically different effects on the magnetic properties of the system. The results may pave the way for the future design of light controllable molecular-scale spintronic devices.
\end{abstract}


Tuning magnetic properties of a material using controllable external methods has been a topic of lasting interest in condensed matter physics and also material science, which has important applications in the field of spintronics. For molecular scale spintronics devices such

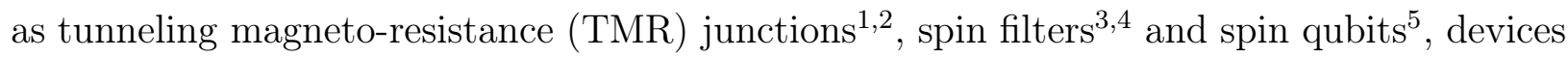
of interest often have ultra small magnetic centers with the size in the order of one nanometer. It is therefore much more challenging to find practical and reliable ways to control the magnetic properties of these systems. In the current study, via first principles calculations, we propose that with the decoration of photochromic molecules, it is possible to use light as an effective external control for the magnetic properties of nanoscale magnetic systems. The combination of transition metal (TM) embedded graphene and the photochromic azobenzene molecule is chosen as the model system for this purpose. We expect our computational studies to stimulate new experiments testing the system we proposed and/or searching for other light-controllable magnetic systems.

The discovery of graphene has spurred vast and growing literatures for the past several years $^{6}$. In this context, there is also a persistent interest to investigate and manipulate magnetic properties of graphene based systems through various means. We are particularly interested in the recent proposal of introducing strongly localized magnetic moments into graphene by embedding TM elements in graphene vacancies ${ }^{7}$. Theoretically, it has been predicted that a range of TM elements embedded in graphene can have magnetic moment from $1 \mu_{B}$ up to more than $3 \mu_{B}$ and these magnetic moments are strongly localized around these embedded TM elements forming molecular scale magnetic centers ${ }^{7-10}$. Experimentally Rodriguez-Manzo et al. demonstrated that scanning transmission electron microscope (STEM) can be used to both image and create vacancies in graphene, which can then be used to deposit metal atoms such as $\mathrm{Fe}, \mathrm{Ni}, \mathrm{Co}$ and $\mathrm{Mo} \stackrel{11}{12}$. By tailoring the temperatures and concentration of the metal atoms, mobility can be controlled to ensure filling of the vacancies. In most cases the vacancies created by the STEM beam are larger than single vacancies $(\mathrm{SV})$ due to the irradiation time and specimen drift during irradiation $\frac{12}{2}$. This observation was also confirmed by Gan et al. who showed that most vacancies in graphene created by ion beams are double or multiple ${ }^{13}$. Wang et al. have also successfully doped a range of metal elements ( $\mathrm{Pt}, \mathrm{Co}, \mathrm{Mn}$ ) into graphene vacancies created with $\mathrm{Au}$ and $\mathrm{B}$ ion bombardments 14 . They noted that there was no contaminations of the ions after the bombardment process, and in turn the vacancies can be doped with the desired metal elements. 
Following these successful works, we chose the graphene with Co embedded in double vacancies (DV) (Fig. 1) for current study since it has been successfully fabricated in different experiments with different techniques.

An azobenzene molecule which basically consists of two benzene rings and two bridging nitrogen atoms, is a photochromic molecule that can transform reversibly between two isomers, namely the trans (Fig. 1(a)) and cis (Fig. 1(b)) isomers upon photo excitation. The energy of the trans isomer is $0.6 \mathrm{eV}$ lower than the cis isomer $\underline{15}$, and the two isomers are separated by an energy barrier of around $1.6 \mathrm{eV} \underline{\underline{16}}$. Both of them are quite stable under room temperature. Transformation from cis to trans can be induced with a laser wavelength of 420 $\mathrm{nm}$, and the opposite way with a laser wavelength of $365 \mathrm{~nm} \frac{17}{}$. The azobenzene derivatives have been proposed in literature to be used as the basis for opto-mechanical devices that can convert the energy of photons to mechanical work ${ }^{17}$ and opto-electronic devices such as light driven molecular switches that can switch on and off electrical current in a circuit $\underline{18-\underline{20}}$. A recent work showed that azobenzene molecules have significant effects on spin polarization of organic-ferromagnetic interfaces. $\stackrel{21}{\underline{1}}$ In this paper, we show by computational studies that it is possible to effectively tune the magnetic properties of a nanoscale system by the decoration of a light-sensitive azobenzene based molecule.

The first principles calculations were performed using density functional theory from the Quantum Espresso package ${ }^{22}$ and we employed generalized gradient approximation (GGA) of Perdew-Burke-Ernzerhof (PBE) format for the exchange and correlation functionals $\underline{23}$. The nuclei and frozen core electrons were modeled with norm conserving ultrasoft pseudopotentials. Kinetic energy cut off was set above $500 \mathrm{eV}$ and each supercell was constructred from $7 \times 7$ graphene unit cell with vacuum space more than $23 \AA$ between graphene layers. The k-point sampling on the graphene plane was $6 \times 6$, and this was found to be sufficient and excellently reproduce prior results for electronic structures and magnetic moments in similar systems with plane-wave basis sets and different choices of GGA functionals -10 . The structure optimization process was performed with force convergence criteria at $0.01 \mathrm{eV} / \AA$. Spin was relaxed in all parts of the calculations.

For Co embedded DV graphene, our calculations gave the C-Co bond length around 1.94 $\AA$ and magnetic moment $1.3 \mu_{B}$ that are almost exactly the same as literature results ${ }^{7}$. Previous results have shown that the magnetic moment comes from one nonbonding $d$ electron of the Co atom $\underline{7}$. Detailed analysis suggested that Co $d_{z^{2}}$ and $d_{x z}$ orbital are almost 


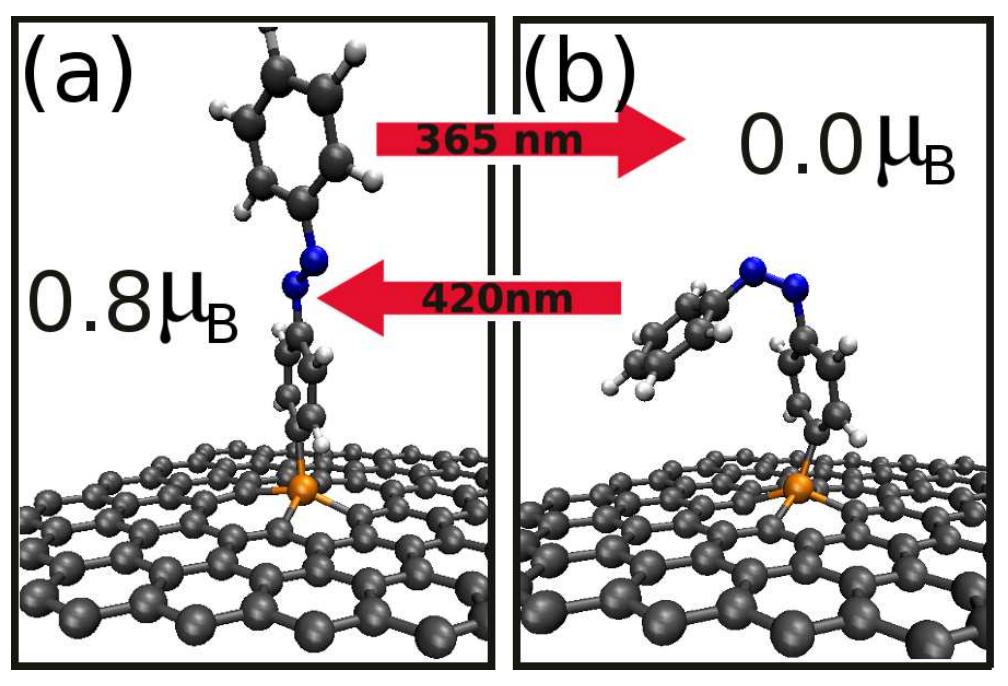

Figure 1: Optimized structures of Co embedded DV graphene with (a) trans and (b) cis azobenzene molecule adsorbed. We first optimized the trans configuration to obtain the structure as shown in (a). The most stable cis configuration was obtained as follows: First, we bent the azobenzene molecule in (a) to be close to its cis isomer, and then relax all degrees of freedom to minimize the total energy. The trans configuration has a magnetic moment of $0.8 \mu_{B}$, and the cis configuration is non-magnetic. The two configurations can transform reversibly into each other upon exposures of laser beam with two different wavelengths as shown in the figure: trans isomer transforms into cis by laser wavelength $\lambda=365 \mathrm{~nm}$, and the reverse by $\lambda=420 \mathrm{~nm}$.

degenerate near Fermi surface. Only one spin channel of the $d_{x z}$ orbital near Fermi surface is occupied, leading to the close-to-one $\mu_{B}$ of magnetic moment. As to azobenzene molecules, our calculations reproduces the geometrical structures in good agreement with prior calculations both in terms of bond lengths and angles to within $1 \%$ accuracy 24 .

We then consider the adsorption of azobenzene molecule on the Co embedded graphene. In order to provide a good contact, one $\mathrm{H}$ atom at the end of the azobenzene is removed. Since the trans isomer is the energetic ground state, we start from the optimization of the adsorption of trans azobenzene. The adsorptions on the Co and also surrounding carbon atoms were tested. It was found that the top site of the Co atom is the mostly preferred. The bonding with the Co atom is more stable by at least $1.2 \mathrm{eV}$ than the bonding with carbon atoms. The most energetically favored adsorption configuration is shown in Fig. 1(a). The C-Co bond length between the molecule and the Co atom in this case is about $1.92 \AA$, and the adsorption energy of the molecule is $2.1 \mathrm{eV}$. The system with this configuration has a 
TRANS

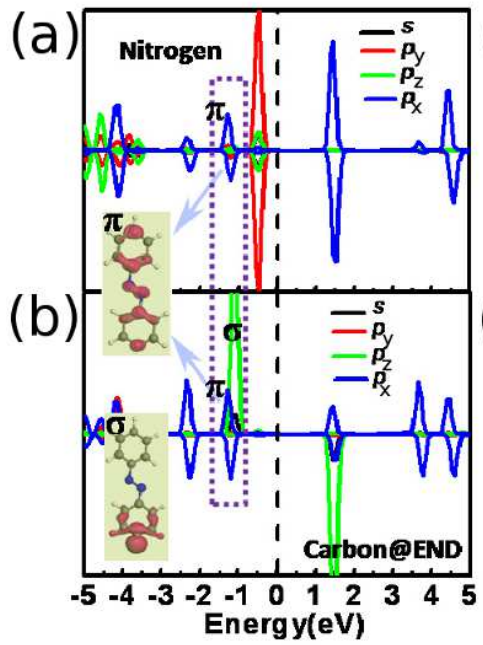

CIS

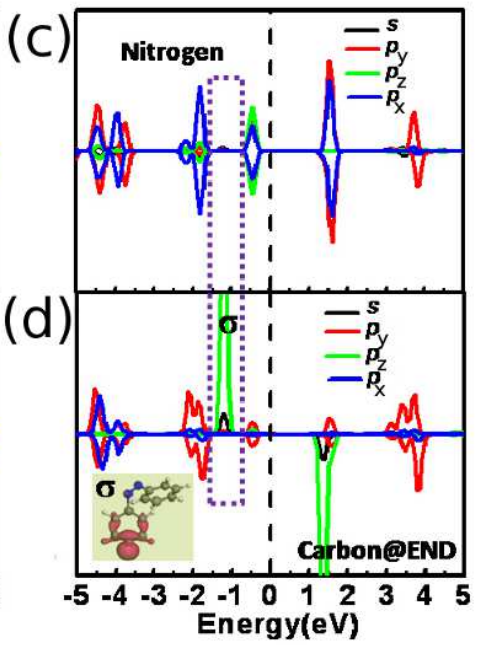

Figure 2: Different electronic structures of two azobenzene isomers after removing one $\mathrm{H}$ atom at end. Spin-polarized density of states of trans isomer projected on (a) briding nitrogen atoms and (b) end carbon atom without hydrogen, and the same for cis configuration in (c) and (d). The isosurfaces of charge density of $\sigma$ and $\pi$-orbital around HOMO are shown in insets respectively. Note that for trans case, there is a $\pi$ character orbital that is energetically degenerate with the dangling HOMO-like $\sigma$ orbital, while in cis, this orbital is missing.

magnetic moment of $0.8 \mu_{B}$, a bit less than the moment without the azobenzene molecule. After the adsorption configuration of trans isomer is obtained, we bend the upper benzene ring of the molecule to be close to the cis isomer, and then relax all degrees of freedom to minimize the total energy to determine the adsorption configuration of the cis isomer, which is actually a process mimicking the photon induced transformation of the molecule. Note that after the optimization, two benzene rings are twisted. The optimized cis adsorption geometry is shown in Fig. 1(b), where the C-Co bond length for cis is about $1.87 \AA$, and the adsorption energy $2.2 \mathrm{eV}$ similar to the trans case. Very interestingly, with the cis molecule, the system is non-magnetic. The drastic change of the magnetic moment from $0.8 \mu_{B}$ for trans adsorption to $0.0 \mu_{B}$ for cis case suggests a reversible light controllable magnetism switching in the system under study.

The magnetism switching is caused by different electronic structures near Fermi surface of two azobenzene isomers, which in turn lead to different interactions between two isomers and the magnetic center, the Co atom in the surface. To see this more clearly, we calculated 
electronic structures of both azobenzene isomers in gas phase and plotted in Fig. 2 the partial density of states (PDOS) projected onto the bridging $\mathrm{N}$ atoms and the end carbon atom of the molecule that forms bond with the Co after adsorption. As one $\mathrm{H}$ atom is removed, one dangling bond of the end $\mathrm{C}$ atom, $\sigma$ orbital, appears as the highest occupied molecular orbital (HOMO) in both cis and trans configurations, occupied by only one electron. For trans there is another $\pi$-character orbital energetically degenerate with the $\sigma$ orbital (Fig. 2(b)). This 'additional' orbital degenerate with HOMO-like $\sigma$ orbital is attributed to the linear configuration of trans isomer in which the $\pi$-orbital of N-N bond (Fig. 2(a)) is delocalized and conjugated with $\pi$-orbital of both benzene rings that can be seen from the isosurface of charge density of this orbital in the inset of the figure. This conjugated $\pi$-orbital is missing in cis isomer due to the bended form of the molecule as we can see from Fig. 2(c) and (d). This difference between trans and cis around HOMO at the end-carbon (without $\mathrm{H}$ ) is crucial for magnetic properties of the azobenzene-decorated system, which will be discussed later.

When the azobenzene molecule adsorbed on Co embedded DV graphene, the halfoccupied $\sigma$-orbital of both trans and cis form $\sigma$ bond with Co atom. This bond is strong enough to stabilize this hybrid system. The zero magnetic moment of the cis configuration can be understood as the fact that after forming a bond with the cis isomer, the originally nonbonding $d$ orbital of the Co atom is satisfied, resulting in a non-magnetic system. The origin of the magnetic moment of the trans configuration is the abovementioned HOMOdegenerate $\pi$-orbital that only exists in trans isomer and the hybridization of Co $d$ orbital and $\mathrm{C} p_{z}$ orbital of trans isomer.

When the Co atom is bonded with azobenzene, the $d_{z^{2}}$ and $d_{x z}$ orbital near the Fermi surface hybridize with the $p_{z}$ orbital of end Carbon atom of the molecule, forming three hybridized orbitals as shown in Fig. 3(c). The Co atom has nine valence electrons. For cis case, one can assume that five of them go into five $\sigma$ C-Co covalent bonds while the left four electrons occupy two lower hybridized states formed by Co $d$ and $\mathrm{C} p_{z}$ orbital as shown in Fig. 3(c). All valencies are saturated and the magnetic moment is zero. However, for trans isomer, as shown in Fig. 2, there is an additional $\pi$ orbital degenerate with HOMO-like $\sigma$ orbital. Electrons of that orbital will also populate to hybridized states (see dashed arrow in Fig. 3(c)) that induces a close-to-one $\mu_{B}$ of magnetic moment. This picture can be easily verified by detailed analysis of electronic structures and magnetic properties. From 

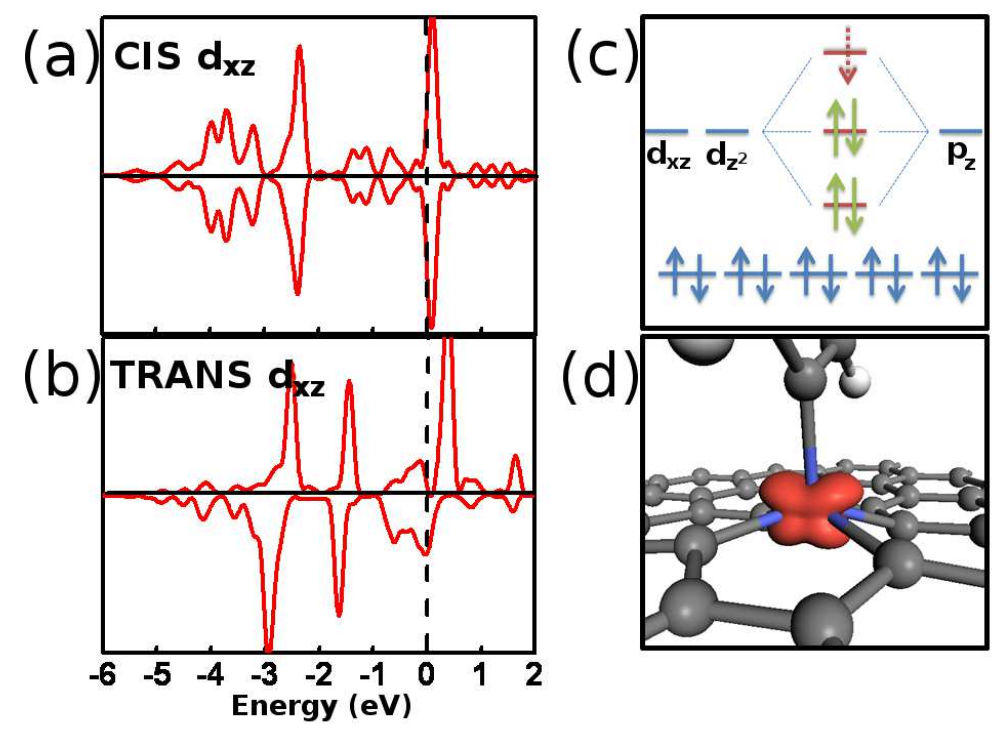

Figure 3: Spin-polarized PDOS of cobalt projected on $d_{x z}$ of (a) cis and (b) trans configurations. In both cases, the $d_{x z}$ orbital split into three peaks after adsorption. Different occupation of the $d_{x z}$ near the Fermi surface lead to different magnetic properties of two configurations. (c) Schematic demonstration of the hybridization of Co $d$ orbitals near Fermi surface with $p_{z}$ orbital of end carbon in azobenzene molecule. Five occupied $\sigma$ orbitals are also shown. After forming these $\sigma$ bonds, the Co atom has 4 leftover valence electrons. For cis case, these four valence electrons occupy two low-lying hybridized orbitals, leaving zero nonbonded electrons and zero magnetic moment. For trans case, electrons in the HOMO-degenerate $\pi$ orbital of azobenzene populates to the highest hybridized orbital (the dashed arrow), resulting in a close-to-one $\mu_{B}$ of magnetic moment. (d) Isosurface of spin density of the system decorated by trans azobenzene. Note the $d_{x z}$ characteristics of the spin density.

the isosurface of spin density of the trans configuration (Fig. 3)(d)), we can see that the magnetic moment localized on the Co atom mainly has characteristics of $d_{x z}$ orbital, and the PDOS projected on Co $d_{x z}$ (Fig. 3(a) and (b)) clearly shows that the orbital splits into three orbitals due to the hybridization in both cases. For cis, both spin-up and spin-down orbitals below Fermi energy are equally occupied and there is one unoccupied orbital right above the Fermi energy for both spin channels, while for trans, for spin-down channel, there are roughly three orbitals occupied, and for spin-up case, only two orbitals occupied, which agree with the aforementioned simple picture.

In conclusion, via first principles methods, we analyzed the electronic and magnetic 
properties of a magnetic graphene-based system decorated with the photochromic dehydrogenated azobenzene molecule. Two isomers of the azobenzene (trans and cis) that can be reversibly converted between each other upon photo excitation are found to have drastically different effects on magnetic properties of the system. These results imply a way to reversibly control the magnetic properties of a nanoscale magnetic system using light, which may have great implications in future applications of molecular spintronic devices. We trust that the similar light-controllable magnetism is can also be found in some other systems, for example, the molecular magnets decorated with photochromic molecules.

We acknowledge the support from Ministry of Education (Singapore) and NUS academic research grants (R-144-000-325-112 and R144-000-298-112). Computational works were performed at the Centre for Computational Science and Engineering parallel computing facilities.

\section{References}

* Electronic address: phyzc@nus.edu.sg

1 C. Zhang, L. L. Wang, H. Cheng, X. Zhang, and Y. Xue, J. Chem. Phys. 124, 201107 (2006).

2 Z. Dai, A. Nurbawono, A. Zhang, M. Zhou, Y. Feng, and C. Zhang, J. Chem. Phys. 134, 104706 (2011).

3 S. Barraza-Lopez, Kyungwha Park, V. Garcá-Suárez, and J. Ferrer, Phys. Rev. Lett. 102, 246801 (2009).

4 M. Zhou, Y. Cai, M. Zeng, C. Zhang, and Y. Feng, J. Chem. Phys. 134, 104706 (2011).

5 T. G. Pedersen, C. Flindt, J. Pedersen, N. Mortensen, A. Jauho, and K. Pedersen Kyungwha, Phys. Rev. Lett. 100, 136804 (2008).

6 A. C. Neto, F. Guinea, N. Peres, K. Novoselov, A. Geim, Rev. Mod. Phys. 81, 109 (2009)

7 A. Krasheninnikov, P. Lethinen, A. Foster, P. Pyykkö, R. Nieminen, Phys. Rev. Lett. 102, 126807 (2009).

8 E. Santos, D. Sánchez-Portal, A. Ayuela, Phys. Rev. B 81, 125433 (2010).

9 E. Santos, A. Ayuela, D. Sánchez-Portal, New J. Phys. 12, 053012 (2010) 
10 J. Kang, H. Deng, S. Li, J. Li, J. Phys.: Condens. Matter 23, 346001 (2011).

11 J. Rodríguez-Manzo, F. Banhart, Nano Lett. 9, 2285 (2009).

12 J. Rodríguez-Manzo, O. Cretu, F. Banhart, ACS Nano 4, 3422 (2010).

13 Y. Gan, L. Sun, F. Banhart, Small 4, 587 (2008).

14 H. Wang, Q. Wang, Y. Cheng, K. Li, Y. Yao, Q. Zhang, C. Dong, P. Wang, U. Schwingenschlögl, W. Yang, X. Zhang, Nano Lett. 12, 141 (2012).

15 F. Schulze, H. Petrick, H. Cammenga, H. Klinge, Zeitschrift für Physikalische Chemie Neue Folge 107, 1 (1977).

16 E. Talaty, J. Fargo, Chem. Commun. 2, 65 (1967).

17 T. Hugel, N. Holland, A. Cattani, 1. Moroder, M. Seitz, H. Gaub, Science 296, 1103 (2002).

18 C. Zhang, M. Du, H. Cheng, X. Zhang, A. Roitberg, J. Krause, Phys. Rev. Lett. 92, 158301 (2004).

19 C. Zhang, Y. He, H. Cheng, Y. Xue, M. Ratner, X. Zhang, P. Krstic, Phys. Rev. B 73, 125445 (2006).

20 M. Valle, R. Guitérez, C. Tejedor, G. Cuniberti, Nature Nano. 2, 176 (2007).

21 Y. Wang, J. G. Che, J. N. Fry, and H. Cheng, J. Phys. Chem. Lett. 4, 3508 (2013).

22 P. Giannozzi, S. Baroni, N. Bonini, M. Calandra, R. Car, C. Cavazzoni, D. Ceresoli, G. Chiarotti, M. Cococcioni, I. Dabo, et al., J. Phys.: Condens. Matter 21, 395502 (2009).

23 J. Perdew, K. Burke, M. Ernzerhof, Phys. Rev. Lett. 77, 3865 (1996).

24 H. Fliegl, H. Köhn, C. ättig, R. Ahlrichs, J. Am. Chem. Soc. 125, 9821 (2003). 